\title{
Wind Turbine Tower Mode Analysis Considering Blade and Centrifugal Stiffening Effect
}

\author{
Wenxing Xiao ${ }^{1}$ and Tao Yang ${ }^{2}$ \\ ${ }^{1}$ China-EU Institute for Clean and Renewable Energy, Huazhong University of Science and Technology, Wuhan 430074, China \\ ${ }^{2}$ School of Energy and Power Engineering, Huazhong University of Science and Technology, Wuhan 430074, China
}

\begin{abstract}
In order to study the influence of wind turbine blade and the blade centrifugal stiffening effect on natural vibration characteristics of wind turbine tower, the finite element model of wind turbine tower was established. By calculating the axial force under the rotating state of the blade, the additional stiffness caused by the centrifugal stiffening effect of the blade was obtained. The natural vibration properties of wind turbine tower structure under three conditions were calculated and the natural frequencies were compared. The results show that the influence of the blade makes the natural frequency of the wind turbine tower structure decreased and the natural frequency of the wind turbine tower structure considering the centrifugal stiffening effect is higher than that not considering the centrifugal stiffening effect. Therefore, it is necessary to consider the influence of the blade and the blade centrifugal stiffening effect on the dynamic characteristics analysis of the wind turbine tower structure.
\end{abstract}

Keywords -wind turbine tower; the centrifugal stiffening effect; modal analysis

\section{INTRODUCTION}

The tower is an elongated thin-walled structure. As the main load-bearing component of a wind turbine, it carries the weight of itself, the rotor, the hub and the nacelle. And it is also in a complex natural environment, subjecting to complex alternating and random loads. So, its own deformation and vibration is very obvious. Therefore, for studying its vibration response under complex wind loads, we must study its own inherent vibration characteristics firstly. The inherent characteristics of the tower are not only affected by its own material, shape, thickness and height, but also by the overhead weight, mass eccentricity. In particular, it is also influenced by the blades and the blade centrifugal stiffening effect in rotating process. Therefore, this paper studies the natural vibration characteristics of the wind turbine tower considering the blade and the blade centrifugal stiffening effect.

In the past, the research on the natural vibration characteristics of wind turbine tower mostly only considered the effect of the tower top mass, the presence or absence of gateways and the foundation stiffness. Zoulong et al studied the influence of the tower top mass on the natural vibration characteristics of the tower [1]. Li Bin et al studied the natural vibration characteristics of two tower structures with holes and without holes [2]. Wang Zhenyu et al studied the influence of the interaction between the foundation and the tower on the natural vibration characteristics of the tower [3]. In summary, there are relatively few studies on the dynamic characteristics of wind turbine tower structures considering the effect of blade and the centrifugal stiffening effect caused by blade rotation.

Based on that, a wind turbine tower model with blades is established in this paper. At the same time, by calculating the axial force under the rotating state of the blade, the additional rigidity caused by the centrifugal stiffening effect of the blade is obtained. In order to study the influence of the blade centrifugal stiffening effect on the natural vibration properties of the wind turbine tower structure, this paper calculates the natural frequency of the wind turbine tower structure in three cases. Case 1 is not taking into account the blade and centrifugal stiffening effect, only the wind wheel and nacelle as a concentrated mass attached to the top of the tower; Case 2 is considering the blade, but not considering the blade centrifugal stiffening effect; Case 3 is considering the blade, and taking into account the centrifugal stiffening effect.

\section{FInITE ELEMENT MODELING OF WIND TURBINE TOWER STRUCTURE}

\section{A. Geometric Parameters}

This paper takes a $5 \mathrm{MW}$ wind turbine as the research object [4]. Tower height is 87.6 meters; the bottom diameter is 6 meters and the thickness is $27 \mathrm{~mm}$; Tower top diameter is 3.87 meters and thickness is $19 \mathrm{~mm}$. The total mass of the tower is $347460 \mathrm{~kg}$. Blade length is 61.5 meters and the quality is 17740 kilograms. Nacelle geometry is $10 \mathrm{~m} \times 5 \mathrm{~m} \times 5 \mathrm{~m}$ and the quality is $240000 \mathrm{~kg}$. The hub mass is $56780 \mathrm{~kg}$. The hub center is $5 \mathrm{~m}$ from the tower axis along the height of the hub. The tower axis is 1.9 meters from the geometric center of the cabin. The top of the tower is 2.4 meters from the hub axis height.

\section{B. Finite Element Modeling}

During the finite element modeling, all parts of the wind turbine are used soild186; Regardless of the bottom opening door of the tower and the interaction with the foundation. In the bottom, all degrees of freedom are restrained; the blade and the hub, the hub and the nacelle, and the nacelle and the tower and the three-section tower are all bonded. The finite element model of wind turbine is shown in Figure I. 


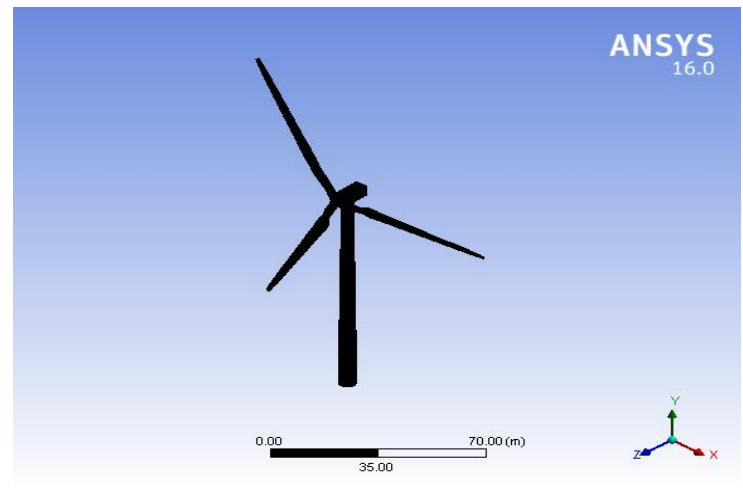

FIGURE I. FINITE ELEMENT MODEL OF WIND TURBINE

\section{BLADE CENTRIFUGAL ST IFFENING EFFECT}

Wind turbine blade will be affected by the axial force during rotation process. The axial force is the composition of the weight of the blade gravity and the centrifugal inertial force along the blade axis. Axial force will make the deformation of the blade and produce additional stiffness that is centrifugal stiffening effect. For the calculation of the additional stiffness, the blade can be analyzed as a variable cross-section rectangular cantilever beam. According to the finite element theory, the blades are discretized into $\mathrm{n}$ discrete points, and the vibration problem of the blade is transformed into the vibration problem of the finite freedom degree system [5].

The multi freedom degree system dynamic equilibrium equation as following:

$$
[M]\{\ddot{x}\}+[C]\{\dot{x}\}+[\bar{K}]\{x\}=\{F(t)\}
$$

$[\mathrm{M}]$ is the mass matrix; $[\mathrm{C}]$ is the damping matrix; $[\bar{K}]$ is the blade stiffness considering the centrifugal stiffening effect, $[\bar{K}]=\left[K_{B}\right]+\left[K_{B G}\right] ;\left[K_{B}\right]$ is the matrix of the blade itself; $\left[K_{B G}\right]$ is an additional stiffness matrix considering the axial force of the blade. As shown in the following formula:

$$
\left[K_{B G}\right]=\left[\begin{array}{cccc}
\frac{N_{1}}{l_{1}} & \frac{-N_{1}}{l_{1}} & \cdots & 0 \\
\frac{-N_{1}}{l_{1}} & \frac{N_{1}}{l_{1}}+\frac{N_{2}}{l_{2}} & \ldots & 0 \\
\vdots & \vdots & \ddots & \frac{-N_{n-1}}{l_{n-1}} \\
0 & 0 & \frac{-N_{n-1}}{l_{n-1}} & \frac{N_{n-1}}{l_{n-1}}+\frac{N_{n}}{l_{n}}
\end{array}\right]
$$

Where $l_{i}$ is the $\mathrm{i}$ unit length; $N_{i}$ is the i-point axial force. During the blade rotation, the axial force changes periodically. so that the blade stiffness also changes. The axial force calculation in the blade rotation process requires the weight and centrifugal inertia force per unit length of the blade. Take the point $\mathrm{i}$ on the blade as an example. This point distance to the center of the shaft is $x_{i}$. The centrifugal forceT $\left(x_{i}\right)$ produced during the rotation of the blade is:

$$
\mathrm{T}\left(x_{i}\right)=0.5 m \Omega^{2}\left(L_{B}^{2}+2 L_{B} L_{R}-2 L_{R} x_{i}-x_{i}^{2}\right)
$$

Where: $m$ is the blade mass per unit length; $\Omega$ is the blade rotation angular velocity; $L_{B}$ is the length of the blade; $L_{R}$ is the blade root to the center of the shaft. During the rotation of the blade, the gravity is periodic and related to the rotation angle of the blade. Assuming that the blade rotation angle is $\theta=\Omega \times$ $\mathrm{t}$, as shown in Figure II. The solid line is the initial position of the blade, and the dotted line is the position where the blade rotates after the angle $\theta$. The component force of blade gravity in the axial direction is:

$$
G_{1 i l}=G_{1 i} \cos \Omega t ; G_{2 i l}=G_{2 i} \sin \left(30^{\circ}+\Omega t\right) ; G_{3 i l}=G_{3 i} \sin \left(30^{\circ}-\Omega t\right)
$$

Where $\mathrm{G}_{1 \mathrm{il}}$ is the component of $\mathrm{i}$ unit gravity $\mathrm{G}_{1 \mathrm{i}}$ along the blade1 axis; $\mathrm{G}_{2 \mathrm{il}}$ is the component of $\mathrm{i}$ unit gravity $\mathrm{G}_{2 \mathrm{i}}$ along the blade 2 axis; $G_{3 i l}$ is the component of $i$ unit gravity $\mathrm{G}_{3 \mathrm{i}}$ along the blade 3 axis.
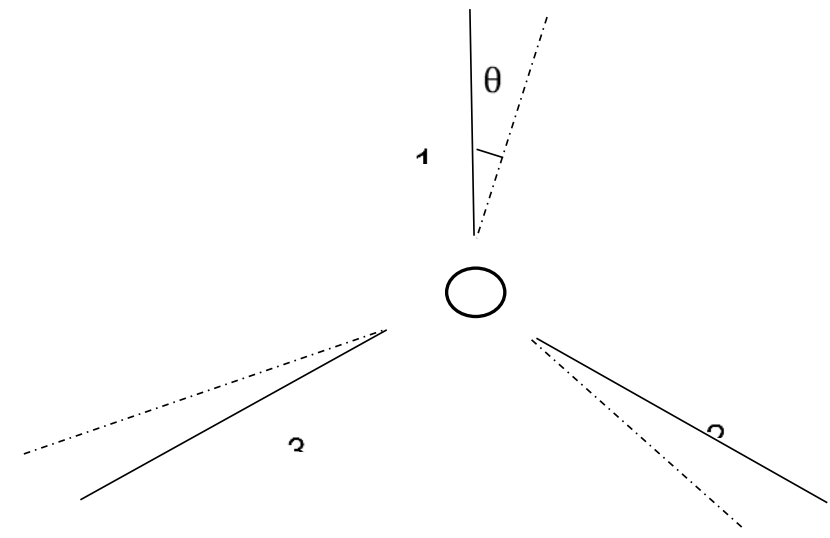

\section{FIGURE II. SCHEMAT IC DIAGRAM of ROT ATIONAL BLADE}

Thus, the resultant force $N_{i}$ at node $i$ on the blade is obtained as follows:

$$
N_{1 i}=\mathrm{T}\left(x_{i}\right)+G_{1 i l} \quad N_{2 i}=\mathrm{T}\left(x_{i}\right)+G_{2 i l} \quad N_{3 i}=\mathrm{T}\left(x_{i}\right)+G_{3 i l}
$$

The axial force of the blade is obtained by the above formula. In the formula (2), an additional stiffnes s matrix can be obtained considering the centrifugal stiffening effect of the blade.

For modal analysis of wind turbine considering blade centrifugal stiffening effect, the calculated axial blade force is used as prestressing force to form additional stiffness matrix in the axial direction of the blades [6].

\section{MODAL ANALYSIS}

In order to study the influence of the blade centrifugal stiffness effect on the natural vibration properties of the wind turbine tower structure, this paper calculates the natural frequency of the wind turbine tower structure in three cases. Case 1 is not taking into account the blade and centrifugal stiffening effect, only the wind wheel, hub and cabin as a concentrated mass attached to the top of the tower; Case 2 is 
considering the blade, but not considering the blade centrifugal stiffening effect; Case 3 is considering the blade, taking into account the centrifugal stiffening effect. The natural frequency distribution of the three cases are shown in Figure III.

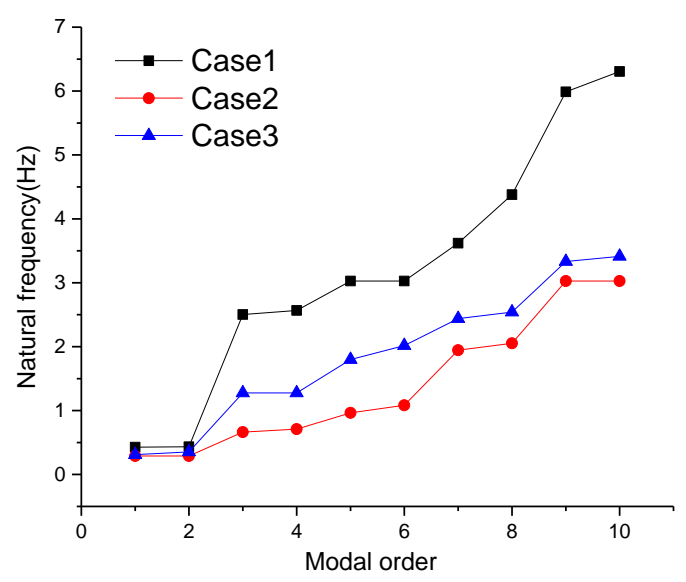

FIGURE III.

NATURAL FREQUENCY DISTRIBUTION

As can be seen from Figure III: The natural frequency of Case 2 is much less than that of Case1, which shows that the blade has a great influence on the natural frequency of wind turbine tower. This may be due to the fact that the overall structure of the wind turbine is dominated by the left and right swinging of the blades and the waving of the blades. The influence of the blades greatly reduces the rigidity of the overall structure of the wind turbine, resulting in a decrease of the natural frequency of the structure. The natural frequency of case 3 is slightly higher than that of Case2, which may be due to the centrifugal stiffening effect caused by the blade rotation increases the blade stiffness, thus enhancing the overall rigidity of the wind turbine tower structure. The natural vibration characteristics show that for the dynamic response analysis of wind turbine tower, we must take into account the influence of blade force and the blade centrifugal stiffening effect.

\section{SUMMARY}

In order to study the influence of blade and blade rotation on the natural vibration characteristics of wind turbine tower structure, a finite element model of wind turbine tower structure with blades was established. At the same time, by calculating the axial force under blade rotation condition, the additional stiffness caused by the blade centrifugal stiffening effect was obtained. Through the modal analysis of wind turbine tower structure of Case1, Case 2 and Case3, it is clear that the centrifugal stiffening effect of blades and blades have a great influence on the inherent properties of wind turbine tower structure. The influence of the blade greatly reduces the natural frequency of the wind turbine tower structure, while the natural frequency of the wind turbine tower considering the blade centrifugal stiffening effect is slightly higher than that only considering stationary blades. This may be due to the centrifugal stiffening effect of the blade increases the blade stiffness, resulting in increase of natural frequency. Therefore, in order to be more realistic, in studying the dynamic characteristics and response of the wind turbine tower structure, it is necessary to consider the influence of the blade and the centrifugal stiffening effect of the blade.

\section{REFERENCES}

[1] ZOU Long. Static and dynamic characteristics of fan tower and fatigue analysis [D]. Three Gorges University, 2014.

[2] LI Bin, JIANG Fu-jie. Modal analysis of wind turbine cone-tower [J]. Journal of Inner Mongolia University of Science and T echnology, 2009, 28 (4): 364-368.

[3] Wang Zhenyu, Zhang Biao, Zhang Zihua, et al.Study on dynamic charact eristics of $1.5 \mathrm{MW}$ wind turbine tower-foundation $[\mathrm{J}]$.Engineering \& Engineering, 2011 (2): 29-36.

[4] Jonkman J, Butterfield S, Musial W, et al. Definition of a 5-MW Reference Wind T urbine for Offshore System Development[J]. Office of Scientific \& T echnical Information Technical Reports, 2009.

[5] Chen Xiaobo, Li Jing, Chen Jianyun. Analysis of Wind-induced Vibration Response of Wind Turbine Tower Considering Leaf Centrifugal St iffness [J] Journal of Engineering Mechanics, 2010, 27 (1): 240-245.

[6] KE Shi-tong, WANG T ong-guang, CAO Jiu-fa, et al.Analysis of windinduced vibration of wind turbine tower considering blade rotation and centrifugal force [J] .Journal of Chinese Journal of Solar Energy, 2015, 36 (1): $33-40$ 